\title{
Predição do Comportamento Térmico de Tubos Compósitos Através de Redes Neurais
}

\author{
Sheila Contant, Liliane M. F. Lona \\ Faculdade de Engenharia Química, UNICAMP, SP
}

Verônica M. A. Calado

Escola de Química, UFRJ

\begin{abstract}
Resumo: Compósitos poliméricos (plásticos reforçados) são materiais formados a partir de um reforço (fase descontínua, normalmente uma fibra) e uma matriz polimérica. Esse tipo de material apresenta várias vantagens em relação aos materiais convencionais de engenharia. Entre os métodos de fabricação de compósitos poliméricos está o filament winding (filamento contínuo ou enrolamento filamentar), um processo empregado na fabricação de sólidos de revolução, como tubos e tanques. Neste trabalho, redes neurais artificiais, uma ferramenta computacional inspirada no funcionamento do cérebro humano, foram aplicadas ao processo de filament winding para predição do comportamento térmico de tubos compósitos durante a etapa de cura. Informações sobre o comportamento térmico das peças compósitas podem auxiliar na seleção do ciclo de cura, que é um dos desafios na obtenção de peças de qualidade e a um baixo custo. As redes neurais foram treinadas com dados obtidos através do modelo Lee-Springer. A metodologia foi validada com resultados experimentais da literatura.
\end{abstract}

Palavras-chave: Compósitos poliméricos, comportamento térmico, redes neurais, modelagem.

\section{Prediction of Thermal Behavior of Composite Tubes Using Neural Networks}

Abstract: Polymeric composites or reinforced plastics are materials made with a polymer matrix and a reinforcement. These materials have many advantages when compared to conventional engineering materials. Among the manufacturing methods for composite parts from continuous fiber reinforcement is the filament winding, often used to fabricate closedsurface structures such as tubes and tanks. In this work artificial neural networks, a computational tool inspired in the human brain, were applied in the filament winding process to predict the thermal behavior of composite tubes during the curing step. Information about the thermal behavior of composite parts may help in the selection of the appropriate cure cycle, which is one of the challenges in obtaining quality parts at low cost. Networks were trained with data obtained with the Lee-Springer model. The methodology was validated with experimental results from the literature.

Keywords: Polymeric composites, thermal behavior, neural networks, modeling.

\section{Introdução}

Compósitos poliméricos (também denominados plásticos reforçados) são materiais formados por uma matriz polimérica e um reforço (fase descontínua, normalmente uma fibra). Entre as vantagens dos compósitos poliméricos estão: baixo peso, resistência à corrosão e a temperaturas elevadas e ótimas propriedades mecânicas, quando comparados aos materiais convencionais de engenharia ${ }^{[1]}$. Entre os métodos de fabricação de compósitos poliméricos de matriz termorrígida está o filament winding (filamento contínuo ou enrolamento filamentar), um processo complexo empregado na fabricação de peças como dutos e tanques, por apresentar baixo custo, altas taxas produtivas e possibilitar a fabricação de várias peças ao mesmo tem$\mathrm{po}^{[2]}$. Esse processo, ilustrado na Figura 1, consiste basica- mente em impregnar um feixe de fibras com uma resina e envolver este conjunto (fibra-resina) através de um molde giratório (mandril), onde se dará a cura. O banho de resina ocorre em uma plataforma que se desloca com uma velocidade conhecida $\mathrm{V}$, cuja relação com a velocidade angular $W$ de rotação do mandril determina a orientação das fibras (ângulo $\phi$ ). Uma tensão $\mathrm{F}$ é aplicada ao reforço à medida que ele é enrolado, produzindo uma pressão de compactação na camada anterior de reforço ${ }^{[3]}$.

Um dos desafios na obtenção de peças de qualidade e a um baixo custo reside na seleção do ciclo de cura (seqüência de temperatura e pressão aplicadas durante o processamento por um determinado tempo), uma vez que este afeta significativamente a performance do produto final ${ }^{[4,5]}$. A escolha do ciclo de cura ótimo não é uma tarefa trivial, uma vez que as reações de cura são acompanhadas de fenômenos complexos

Autor para correspondência: Liliane M. F. Lona, Faculdade de Engenharia Química, UNICAMP, Caixa Postal 6066, CEP:13081-970, Campinas, SP. E-mail: liliane@feq.unicamp.br 


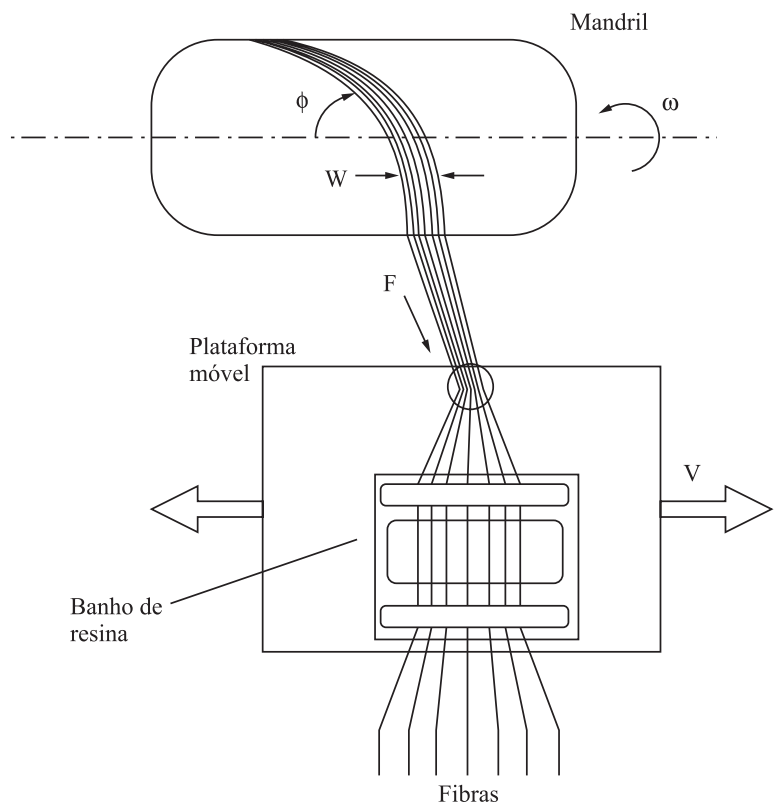

Figura 1. Esquema do processo de filament winding ${ }^{[3]}$

que ocorrem no material compósito, os quais ainda não são totalmente compreendidos ${ }^{[6,7]}$.

Neste trabalho, propõe-se a modelagem do processo de filament winding através de redes neurais, uma técnica computacional inspirada no funcionamento do cérebro humano. Essa técnica mostra-se bastante interessante devido a sua habilidade para aprender o comportamento de um processo e as relações entre grupos de variáveis sem a necessidade de um modelo fenomenológico do sistema. Além disso, é capaz de trabalhar com problemas de elevada complexidade, não lineares, de larga escala e mesmo os considerados intratáveis, apresentando um tempo de processamento reduzido se comparado aos métodos tradicionais ${ }^{[8,9]}$. A literatura mostra diversos trabalhos recentes que comprovam a eficiência das redes neurais no estudo de problemas da área de materiais, incluindo os materiais compósitos ${ }^{[10]}$.

Uma rede neural típica consiste de elementos de processamento (neurônios) distribuídos em três tipos distintos de camadas e conectados através de canais de fluxo de informações denominados pesos. O treinamento (ou fase de aprendizado da rede) consiste basicamente em um ajuste desses pesos. Redes neurais foram empregadas neste trabalho na predição da variação de temperatura no interior de peças durante a etapa de cura, sendo os dados de treinamento obtidos através do modelo LeeSpringer ${ }^{[11]}$. Esse modelo resolve o balanço diferencial de energia e leva em conta a reação de cura da matriz polimérica.

\section{Metodologia para Treinamento das Redes}

\section{Dados Utilizados}

Os dados para treinamento das redes foram obtidos através do software Windthick que é baseado no modelo LeeSpringer ${ }^{[11]}$ e considerando dois tipos de resina epóxi (Fiberite 976 e HBRF55) e dois tipos de fibra (grafite Thornel T-300 e fibra de vidro tipo $S$ ), cujas propriedades são mostradas nas
Tabela 1. Propriedades das resinas

\begin{tabular}{lcc}
\hline \multicolumn{1}{c}{ Propriedade } & Fiberite 976 $^{[15]}$ & HBRF55 $^{[16]}$ \\
\hline Densidade $\left(\mathrm{kg} / \mathrm{m}^{3}\right)$ & 1260 & 1261,6 \\
Calor específico (kJ/kg.K) & 1,26 & 1,34 \\
Condutividade térmica (J/s.m.K) & 0,167 & 0,25 \\
\hline
\end{tabular}

Tabela 2. Propriedades das fibras

\begin{tabular}{|c|c|c|}
\hline Propriedade & Grafite T-300 ${ }^{[15]}$ & S-Glass ${ }^{[13]}$ \\
\hline Densidade $\left(\mathrm{kg} / \mathrm{m}^{3}\right)$ & 1790 & 2491 \\
\hline Calor específico $(\mathrm{kJ} / \mathrm{kg} . \mathrm{K})$ & 0,712 & 0,712 \\
\hline $\begin{array}{l}\text { Condutividade térmica } \\
\text { transversa }(\mathrm{J} / \mathrm{s} \cdot \mathrm{m} . \mathrm{K})\end{array}$ & 26,0 & 3,03 \\
\hline $\begin{array}{l}\text { Condutividade térmica } \\
\text { longitudinal (J/s.m.K) }\end{array}$ & 8,5 & 3,03 \\
\hline
\end{tabular}

Tabelas 1 e 2, respectivamente. Foram simulados 21 tubos com fração de volume de fibras entre 50 e $70 \%$ e espessuras da peça entre 0,15 e 1,2 in. Diferentes ciclos de cura foram assumidos (temperatura aplicada, taxa de aquecimento e resfriamento e tempo de cura). Para todas as peças, assumiu-se padrão de enrolamento de fibra helicoidal (ângulo de enrolamento $\pm 55^{\circ}$ ), velocidade angular do mandril 3,14 rad/s, diâmetro interno 2" e comprimento 18". Considerou-se um mandril de alumínio com as propriedades mostradas em Calius et alii $(1990)^{[12]}$.

\section{Treinamento das Redes}

Foi empregado o algoritmo de backpropagation ${ }^{[8]}$. A rede contou com sete neurônios na camada input (densidade, condutividade térmica e calor específico da camada compósita, espessura da peça, temperatura na superfície da peça, altura da medição e tempo). Para cálculo das propriedades do compósito, foram utilizadas as expressões propostas em Chamis (1984) ${ }^{[13]}$. Um programa computacional previamente desenvolvido ${ }^{[14]}$ foi utilizado para simulação do processo.

Para o trabalho com as redes neurais, foram consideradas três etapas chamadas de treinamento, teste e predição. Inicialmente, a rede neural foi treinada e testada, simultaneamente. Os erros entre os valores desejados e os valores gerados pela rede para o conjunto de teste serviram com critério de parada para o treinamento da rede. Concluídas essas duas etapas, foi selecionado um novo conjunto de dados (resultados expe-

Tabela 3. Algumas características dos cilindros ${ }^{[12]}$

\begin{tabular}{ccccc}
\hline Cilindro & $\mathbf{D}_{\mathbf{i}}$ (in) & $\phi$ & $\mathbf{L}(\mathbf{i n})$ & $\mathbf{t}(\mathbf{h})$ \\
\hline $\mathrm{A}$ & 6 & $\pm 55^{\circ}$ & 25 & 10 \\
$\mathrm{~B}$ & 3 & $\pm 55^{\circ}$ & 18 & 10 \\
$\mathrm{C}$ & 3 & $\pm 43^{\circ}$ & 20 & 10 \\
$\mathrm{D}$ & 3 & $\pm 40^{\circ}$ & 18 & 14 \\
\hline
\end{tabular}

$\mathrm{D}_{\mathrm{i}}=\overline{\text { diâmetro interno; } \phi \text { = ângulo de enrolamento; } \mathrm{L}=\text { comprimento; } \mathrm{t}}=$ tempo de cura 


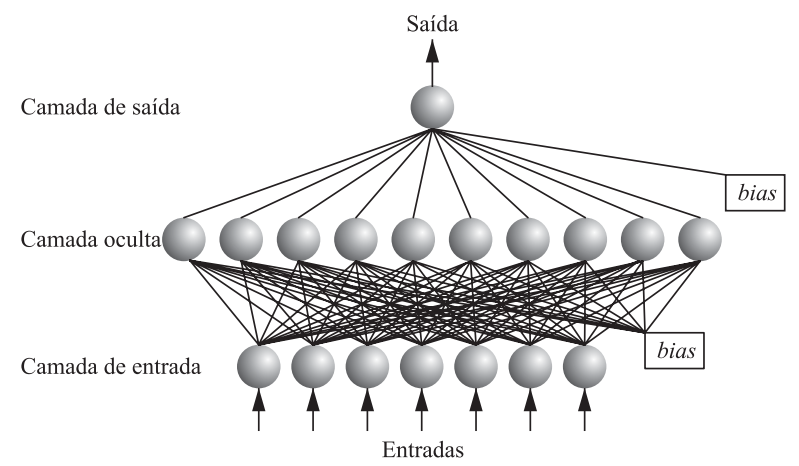

Figura 2: Estrutura da rede neural utilizada: camada de entrada com sete neurônios, camada oculta com dez neurônios e camada de saída com um neurônio (temperatura). Além dos pesos, também foram determinados os bias

rimentais da literatura ${ }^{[12]}$ ), para novos tubos cujas características são mostradas na Tabela 3. Utilizando-se os pesos obtidos durante a etapa de treinamento, a rede neural realizou predições para as_entradas correspondentes aos valores experimentais da literatura. Os resultados fornecidos pela rede neural foram comparados aos resultados experimentais da literatura. Essa última etapa foi denominada etapa de predição.

\section{Resultados e Discussão}

Nesse trabalho, foram utilizadas redes neurais formadas por três camadas (camada de entrada, oculta e de saída). Na camada oculta, foram utilizados 10 neurônios. Na camada de saída, há somente um neurônio, que é a temperatura no interior do tubo em diferentes posições ao longo da sua espessura. Foram utilizados diferentes valores de temperatura no interior do tubo em função da sua espessura (150 valores no conjunto de treinamento e 24 valores no conjunto de teste). Além dos pesos, foram determinados também os bias, para as camadas oculta e de saída, sendo o valor das entradas dos bias igual a 1. A Figura 2 ilustra a rede neural utilizada.

A Figura 3 ilustra a evolução dos erros de treinamento e teste, até 4000 iterações. A escolha da melhor combinação dos parâmetros desse algoritmo foi realizada automaticamente através do programa computacional desenvolvido, que repetiu o treinamento da rede muitas vezes. A Figura 4 compara os valores desejados com os valores gerados pela rede neural. Os valores foram gerados utilizando-se os pesos correspondentes ao menor erro de teste, que, conforme mostra a

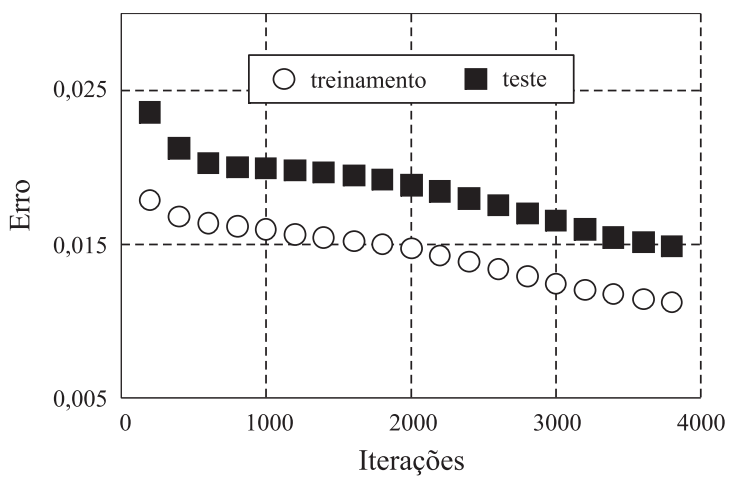

Figura 3. Perfis dos erros de treinamento e teste da rede neural
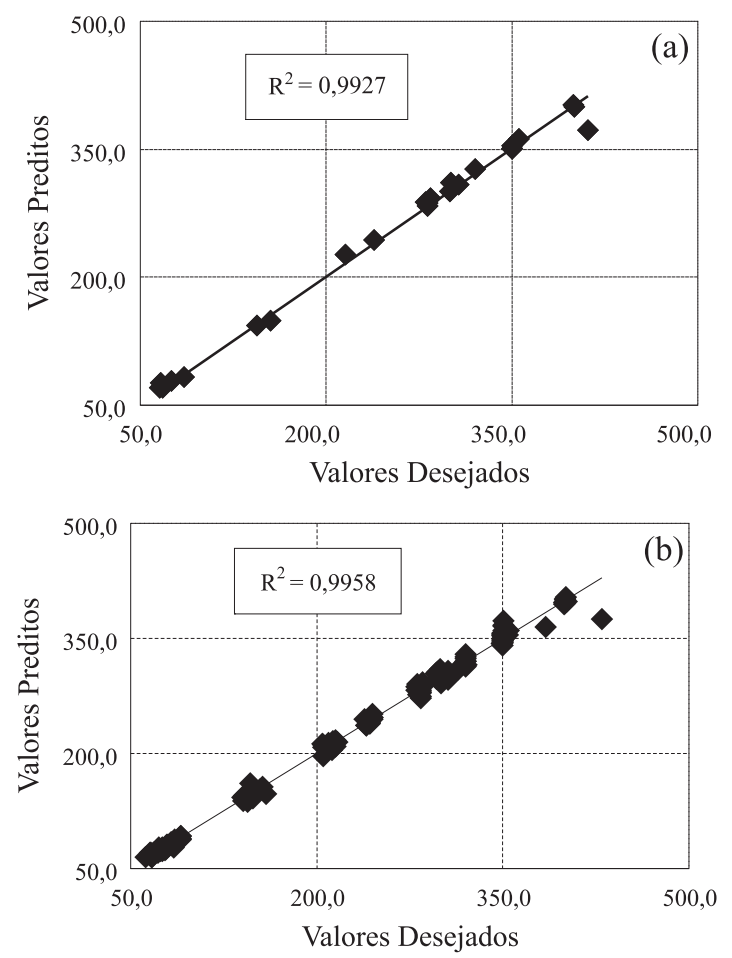

Figura 4. Comparação entre os valores de temperatura desejados e gerados pela rede neural para os conjuntos de: (a) teste e (b) treinamento

Figura 3, aconteceu em 4000 iterações. Os coeficientes de determinação encontrados para os conjuntos de treinamento $\left(\mathrm{R}^{2}=0,9958\right)$ e de teste $\left(\mathrm{R}^{2}=0,9927\right)$ mostram que o aprendizado da rede foi bastante eficiente.

Utilizando os pesos obtidos durante a fase de treinamento, foram obtidos perfis de temperatura, em diferentes pontos da peça, para quatro cilindros mostrados em Calius et alii $(1990)^{[12]}$. Essa etapa foi denominada etapa de predição. Os tubos foram fabricados com resina epóxi Fiberite 976 e fibra de grafite T-300, cujas propriedades são mostradas nas Tabelas 1 e 2, respectivamente.

Para o treinamento e teste da rede foram utilizados sempre dados obtidos para tubos de diâmetro interno de 2" e comprimento de 18 ". Na Tabela 3, onde são apresentados os cilindros utilizados na etapa de predição, os seus diâmetros são diferentes, 3 e 6" e o comprimento de 18 a 25", mesmo assim, obteve-se boas predições. Por apresentarem características diferentes dos tubos dos conjuntos de treinamento e teste, esses novos dados não foram incluídos na etapa de teste, mas sim na etapa de predição.

Com relação ao comprimento dos tubos, o modelo que forneceu os dados para treinamento da rede assume que para cilindros longos (aqueles para os quais o comprimento é muito maior que a espessura), os gradientes axiais de temperatura podem ser desprezados. Resultados experimentais reportados na literatura ${ }^{[12]}$ sugerem que as variações de temperatura ao longo do comprimento de tubos "finos" podem ser consideradas desprezíveis e resultados numéricos da literatura $^{[12]}$ mostram que esses gradientes de temperatura podem ser desprezíveis mesmo para cilindros espessos. Portanto a utilização de tubos de comprimentos diferentes não interfere 
de maneira significativa na temperatura de cura do compósito. Por outro lado, a temperatura no interior da peça pode ser influenciada por outros fatores, entre eles o diâmetro dos tubos. Em um modelo convencional, cada variável utilizada tem um impacto significativo na resposta e se essa variável é indefinida ou apresenta erro, o modelo poderá gerar resultados incoerentes. As redes neurais, por outro lado, podem trabalhar com dados numéricos incompletos, inconsistentes e imprecisos e mesmo se o valor de uma variável não é definido, a resposta poderá não ser substancialmente afetada ${ }^{[9]}$.

Os resultados obtidos com as redes neurais foram comparados com os resultados experimentais reportados na literatura e são mostrados na Figura 5 (conjunto de predição). Além da diferença entre os tipos de cilindro, as diferenças de comporta-
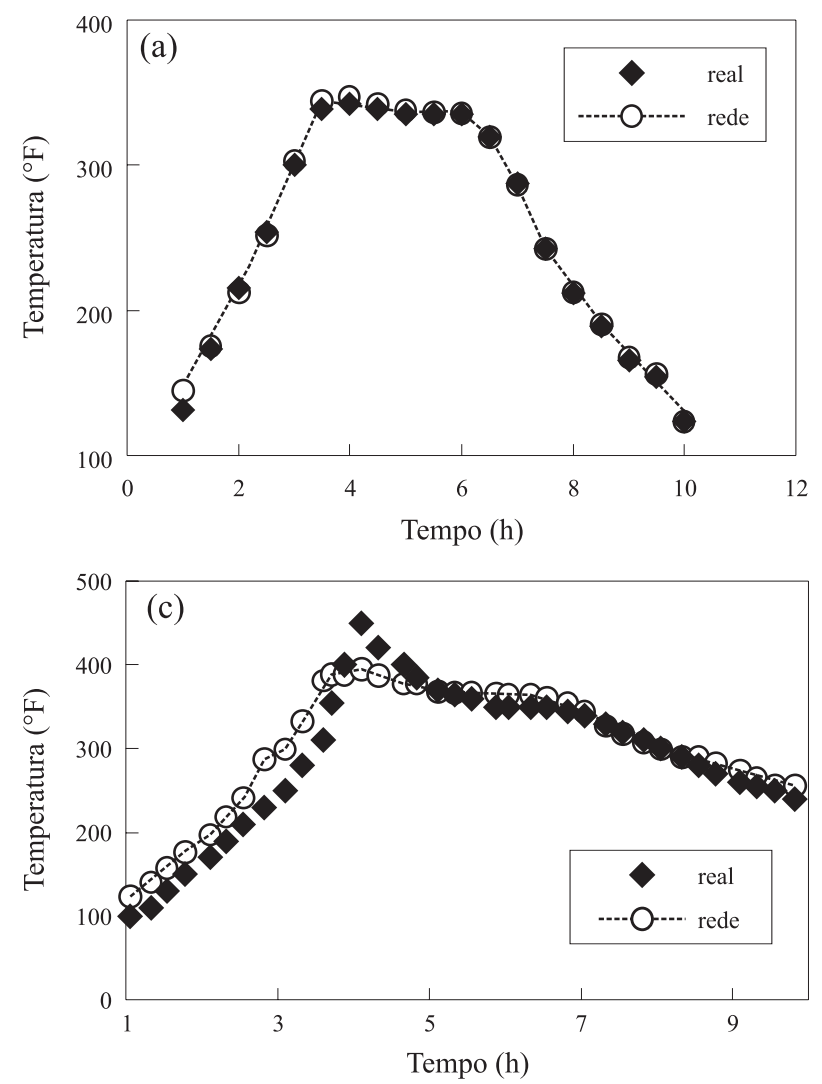

mento observadas de um cilindro para o outro se devem também aos diferentes ciclos de cura aplicados (a temperatura da superfície da peça foi uma variável de entrada da rede neural).

A Figura 6 mostra uma ampliação do pico de temperatura da Figura 5 (e), para permitir uma visão mais clara do que acontece com os resultados da rede para esse pico de temperatura.

Observa-se uma concordância entre os resultados gerados pela rede neural e os valores experimentais para todos os cilindros, em especial para os cilindros $\mathrm{A}$ e B. Com relação às peças $\mathrm{C}$ e D, os desvios observados para alguns pontos podem estar associados ao fato de, para essas peças, as temperaturas medidas na camada mais externa diferirem das temperaturas medidas na superfície mais interna do mandril.
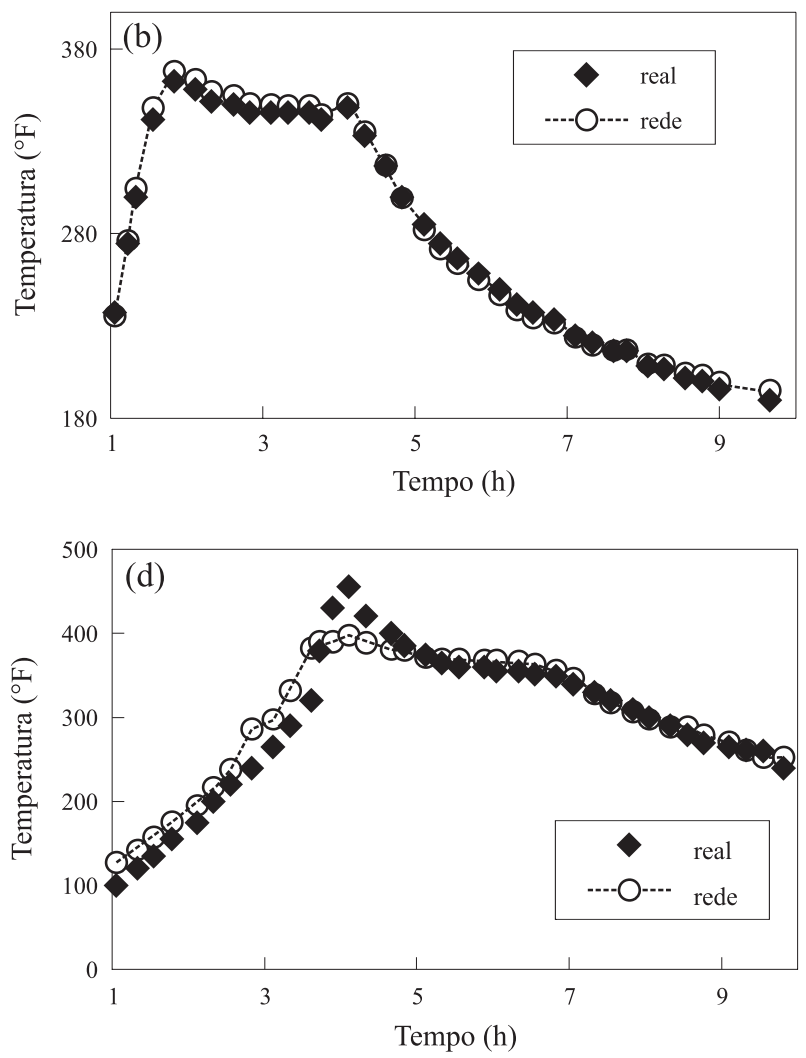

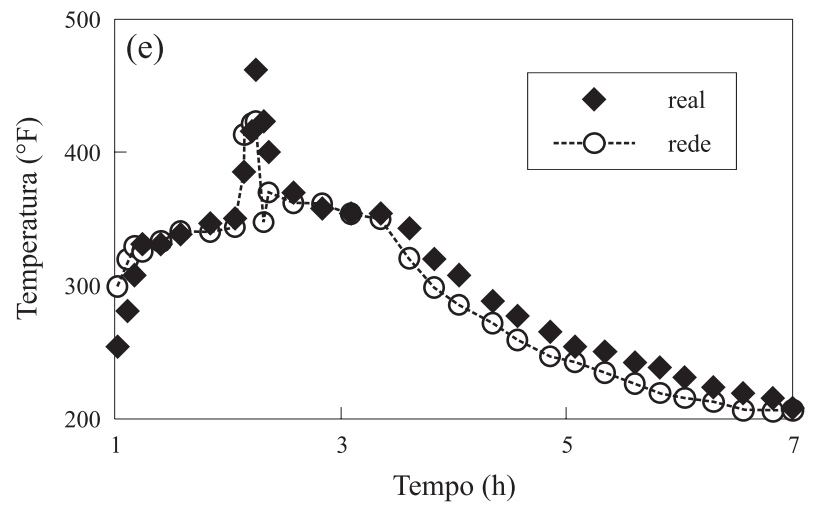

Figura 5. Comparação entre os valores de temperatura gerados pela rede neural e os valores experimentais reportados na literatura ${ }^{[12]}$ referentes à etapa de predição para: (a) cilindro A na metade da peça, (b) cilindro B na camada inferior da peça, (c) cilindro C na metade da peça, (d) cilindro C a 3/4 da superfície externa da peça e (e) cilindro D na metade da peça 


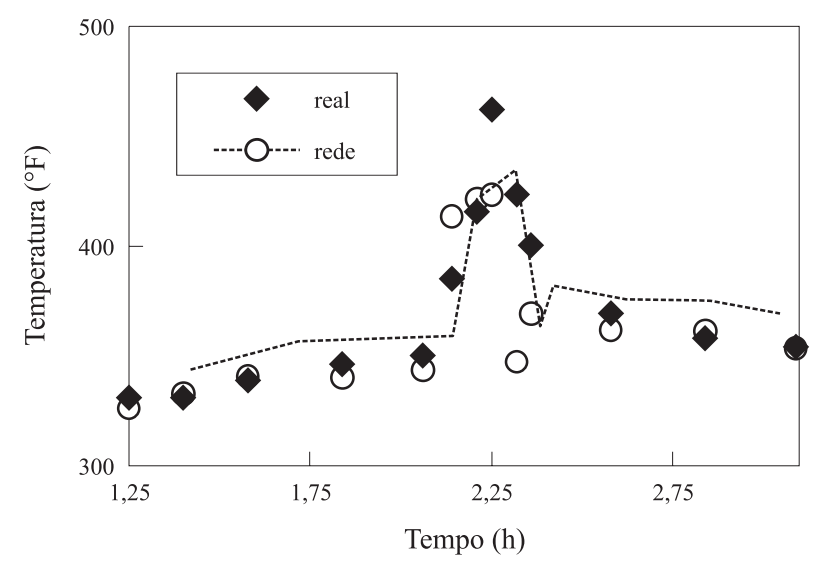

Figura 6. Ampliação do pico de temperatura da Figura 5 (e)

Esses dois conjuntos de temperatura devem ser especificados no software Windthick como condição de contorno e foram considerados como sendo iguais. Embora essa suposição possa não ser realista, não havia nenhuma informação sobre o perfil de temperatura do mandril e, ao invés de utilizar valores arbitrários para a temperatura nessa posição, optou-se por mantê-la igual à temperatura na superfície da peça. Ainda assim, os perfis obtidos pela rede neural são bastante próximos dos obtidos experimentalmente.

Conhecendo-se os valores de temperatura no interior da peça, pode-se ajustar os valores das variáveis de processo de forma a manter a temperatura uniforme em todos os pontos no interior do material e abaixo do máximo valor permitido durante todo o tempo de processamento. Além disso, através desses valores de temperatura é possível obter informações sobre grau de cura no interior da peça. Com isso, pode-se ajustar as condições operacionais para que a peça seja completamente curada e de maneira uniforme, sendo o tempo de processamento o menor possível (seleção do ciclo de cura).

Os modelos cinéticos mais usuais para a cura da resina são o modelo de ordem $n$ e o modelo autocatalítico ${ }^{[7]}$. Para as resinas consideradas nesse trabalho, o grau de cura pode ser definido pelo modelo autocatalítico dado pelas Equações 1 a 4. Os parâmetros dessas equações são mostrados na Tabela 4. As reações de cura que ocorrem nesse processo tem efeitos térmicos significativos, o que levou aos picos de temperatura que aparecem na Figura 5.

$$
\begin{aligned}
& \frac{d \alpha}{d t}=\frac{H_{T}}{H_{u}}\left(k_{1}+k_{2} \psi^{a}\right)(B-\psi)^{b}\left(1-\psi^{d}\right)^{c} \\
& \psi=\frac{H_{u}}{H_{T}} \int_{0}^{t} \frac{d \alpha}{d t} d t \\
& k_{1}=A_{1} \exp \left(-E_{1} / R T\right) \\
& k_{2}=A_{2} \exp \left(-E_{2} / R T\right)
\end{aligned}
$$

Através das redes neurais, é possível obter a temperatura para um determinado tempo. Utilizando-se essa informação, é possível aplicar as equações anteriores e obter o grau de
Tabela 4. Parâmetros do modelo de cinética de cura das resinas utilizadas ${ }^{[12]}$

\begin{tabular}{ccc}
\hline Parâmetro & HBRF55 & Fiberite 976 \\
\hline$A_{1}$ & $490,6 \mathrm{~s}^{-1}$ & $4400 \mathrm{~s}^{-1}$ \\
$A_{2}$ & $2052,4 \mathrm{~s}^{-1}$ & $7500 \mathrm{~s}^{-1}$ \\
$E_{1}$ & $4,47 \times 10^{4} \mathrm{~J} / \mathrm{mol}$ & $6,25 \times 10^{4} \mathrm{~J} / \mathrm{mol}$ \\
$E_{2}$ & $4,28 \times 10^{4} \mathrm{~J} / \mathrm{mol}$ & $5,68 \times 10^{4} \mathrm{~J} / \mathrm{mol}$ \\
$B$ & 1,065 & 1,0 \\
$a$ & 0,840 & 1,03 \\
$b$ & 0,54 & 1,22 \\
$c$ & 1,0 & 0 \\
$d$ & 1,0 & $0,0044 \mathrm{~T}-1,1 \mathrm{para}$ \\
$H_{T} / H_{u}$ & 1,0 & $\mathrm{~T}<480 \mathrm{~K}$ \\
\hline
\end{tabular}

cura. Em um trabalho futuro, pretende-se utilizar as redes neurais para predição também do grau de cura.

As redes neurais podem requerer, dependendo do processo, um longo tempo para serem treinadas. Porém, uma vez treinadas, elas são capazes de calcular rapidamente resultados a partir de novas entradas, sendo essa uma das grandes vantagens da técnica ${ }^{[9]}$. Nesse trabalho, o treinamento da rede demorou menos de $2 \mathrm{~s}$, para 4000 iterações. A rede neural treinada é capaz de gerar novos resultados de temperatura instantaneamente, uma vez que, conhecidos os pesos, é preciso realizar apenas alguns cálculos simples, que consistem na etapa forward do algoritmo de backpropagation ${ }^{[8]}$. Uma simulação com o Windthick nas mesmas condições de processo simulado e de computador utilizado pode demorar cerca de 10 min (é importante observar que esse software realiza outras predições além da temperatura, o que aumenta o tempo computacional). $\mathrm{O}$ processamento computacional mais rápido das redes neurais pode ser particularmente útil em aplicações que requeiram simulações repetidas, tais como a otimização do ciclo de cura. Além do processamento mais rápido, com a rede neural é possível obter novos resultados para várias condições de entrada ao mesmo tempo (o modelo convencional trabalha com somente uma condição por vez).

\section{Conclusões}

O uso dos compósitos poliméricos - materiais formados por uma matriz polimérica e um reforço, normalmente uma fibra - vem crescendo nas últimas décadas, devido as suas muitas vantagens em relação aos materiais convencionais de engenharia. Neste trabalho, redes neurais foram utilizadas para predição das mudanças de temperatura no interior de peças fabricadas por filament winding. Os dados de treinamento foram obtidos através do modelo Lee-Springer para cilindros de matriz termorrígida. Os resultados encontrados mostraram a eficiência da metodologia proposta, uma vez que 
foram obtidos valores de coeficiente de determinação $\left(\mathrm{R}^{2}\right)$ próximos de 1. Utilizando-se os valores dos pesos obtidos com o aprendizado das redes, foram realizadas predições para novos dados da literatura.

Os resultados aqui apresentados representam a primeira etapa de um trabalho envolvendo redes neurais e compósitos poliméricos; em uma publicação futura, serão mostrados novos resultados envolvendo, entre outros aspectos, a variação das condições operacionais de obtenção dos tubos (velocidade angular, ângulo de enrolamento, etc), o que certamente dará uma melhor visibilidade da aplicação das redes.

Aqui no Brasil, muitas indústrias foram e estão sendo implantadas com o objetivo de produzir os mais variados tipos de peças de material compósito. Apesar da América do Sul representar atualmente apenas 3\% do mercado global de compósitos poliméricos, o crescimento anual do setor nessa região tem sido o maior de todo o mundo ${ }^{[14]}$, demonstrando o imenso espaço que existe para esses materiais. Sendo o Brasil o líder desse mercado na América do Sul, percebe-se a potencialidade deste setor produtivo e a necessidade de se aumentar a sua competitividade, através de pesquisas e aprimoramento de recursos humanos.

\section{Símbolos}

$$
\begin{aligned}
& a=\text { parâmetro cinético } \\
& A_{1}=\text { fator pré-exponencial } \\
& A_{2}=\text { fator pré-exponencial } \\
& B=\text { parâmetro cinético } \\
& b=\text { parâmetro cinético } \\
& c=\text { parâmetro cinético } \\
& d=\text { parâmetro cinético } \\
& \mathrm{D}_{\mathrm{i}}=\text { diâmetro interno } \\
& E_{1}=\text { energia de ativação } \\
& E_{2}=\text { energia de ativação } \\
& \mathrm{F}=\text { tensão } \\
& \mathrm{L}=\text { comprimento } \\
& R=\text { constante dos gases } \\
& T=\text { temperatura } \\
& \mathrm{t}=\text { tempo } \\
& \mathrm{V}=\text { velocidade } \\
& W=\text { velocidade angular de rotação do mandril }
\end{aligned}
$$

\section{Letras Gregas}

$\alpha=$ grau de cura

$\phi=$ ângulo de enrolamento

\section{Agradecimentos}

Os autores agradecem à FAPESP (Proc. 00/10090-7).

\section{Referências Bibliográficas}

1. Agarwal, B. D. \& Broutman, L. J. - "Analysis and Performance of Fiber Composites”, John Wiley \& Sons, EUA (1990).

2. Bannister, M. - Composites: Part A, 2, p.901 (2001).

3. Banerjee, A; Sun, L.; Mantell, S. C. \& Cohen, D. Composites: Part A, 29, p.251 (1998).

4. Loos, A. C. \& Springer, G. S. - J. Comp. Mat., 17, p.135 (1983).

5. Lee, S. \& Springer, G. S. - J. Comp. Mat., 22, p.15 (1988).

6. Gorovaya, T. A. \& Korotkov, V. N. - Composites Part A, 27A, p.953 (1996).

7. Calado, V. M. A. \& Advani S. G. - "Thermoset Cure Kinetics and Rheology", in: Processing of Continuos Fiber Reinforced Composites, R. Davé, A. Loos (ed.), cap. 2, Hanser Publisher, EUA (1999).

8. Haykin, S. - "Redes Neurais, Princípios e Prática", Bookman, Porto Alegre (2001).

9. Baughman, D. R. \& Liu, Y. A. - "Neural Networks in Bioprocessing and Chemical Engineering", Academic Press, EUA (1995).

10. Sumpter, B. G. \& Noid, D. W. - Annual Rev. Mat. Sci., 26, p.223 (1996).

11. Lee, S. \& Springer, G. S. - J. Comp. Mater., 24, p.1270 (1990).

12. Calius, E. P. \& Springer, G. S. - Int. J. Solids Struct., 26, p.271 (1990).

13. Chamis, C. C. - SAMPE Quarterly, abril, p.14 (1984).

14. Contant, S. - “Aplicação de Redes Neurais no Processo de Filament Winding", Tese de Mestrado, Universidade Estadual de Campinas, Brasil (2002).

15. Tang, J.-M.; Lee, W. I. \& Springer, G. S. - J. Comp. Mater., 21, p.421 (1987).

16. Zhao, L.; Mantell, S. C.; Cohen, D. \& McPeak, R. - Comp. Struct., 52, p.499 (2001).

Enviado: $10 / 10 / 03$

Reenviado: $17 / 06 / 04$

Aprovado: 10/09/04 